WellBeing International

WBI Studies Repository

$11-10-2011$

\title{
Learned Recognition and Avoidance of Invasive Mosquitofish by the Shrimp, Paratya australiensis
}

\author{
Joshua D. Bool \\ Macquarie University \\ Kristen Whitcomb \\ Macquarie University \\ Erin Kydd \\ Macquarie University \\ Culum Brown \\ Macquarie University
}

Follow this and additional works at: https://www.wellbeingintlstudiesrepository.org/acwp_asie

Part of the Animal Studies Commons, Behavior and Ethology Commons, and the Comparative Psychology Commons

\section{Recommended Citation}

Bool, J. D., Witcomb, K., Kydd, E., \& Brown, C. (2011). Learned recognition and avoidance of invasive mosquitofish by the shrimp, Paratya australiensis. Marine and Freshwater Research, 62(10), 1230-1236.

This material is brought to you for free and open access by WellBeing International. It has been accepted for inclusion by an authorized administrator of the WBI Studies Repository. For more information, please contact wbisr-info@wellbeingintl.org.

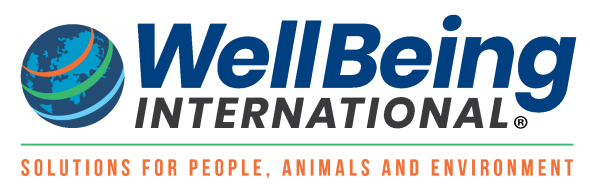




\title{
Learned Recognition and Avoidance of Invasive Mosquitofish by the Shrimp, Paratya australiensis
}

Joshua D. Bool, Kristen Witcomb, Erin Kydd, and Culum Brown

Macquarie University

KEYWORDS

conditioning, crustacean, Gambusia, learning, predator, shrimp

\begin{abstract}
Little is known about the learning ability of crustaceans, especially with respect to their anti-predator responses to invasive species. In many vertebrates, anti-predator behaviour is influenced by experience during ontogeny. Here, predator-naïve glass shrimp (Paratya australiensisis) were exposed to a predatory, invasive fish species, Gambusia holbrooki, to determine whether shrimp could learn to: (1) avoid the scent of Gambusia via classical conditioning; and (2) restrict their activity patterns to the night to reduce predatory encounters. Conditioned shrimp were placed in containers in aquaria containing Gambusia for 3 days during which time they could be harassed but not consumed by Gambusia. When tested in a Y-maze, conditioned shrimp showed a long delay before making a choice between Gambusia scented water and aged tap water but chose an arm at random. Control shrimp showed a brief delay in emergence and also chose at random. In a second experiment, we housed shrimp with a single Gambusia and observed their activity patterns. In the presence of Gambusia, shrimp switched from diurnal to nocturnal foraging. These results show that naïve shrimp learn to recognise novel predatory species via chemical cues and adjust their activity patterns to coincide with periods when Gambusia are inactive.
\end{abstract}

\section{Introduction}

Anti-predator behaviour is anticipated to have a significant innate component because young animals are often highly vulnerable to predation and failure to escape has obvious consequences. However, it is becoming increasingly apparent that a wide variety of animals, including fishes, refine their antipredator behaviour during ontogeny via learning (Brown et al. 2011; Kelley and Magurran 2011). Furthermore, innate and learned components of anti-predator behaviour can interact. Although both predator-naïve and sympatric populations learn to respond to predators, the greatest improvement has been shown in fish derived from predator-sympatric populations (Magurran 1990). Similarly, basic anti-predator behaviours in guppies were inherited and built upon early in life through experience (Magurran and Seghers 1990). Behavioural plasticity brought about through learning is particularly important when animals respond to novel cues, such as the appearance of invasive, predatory species. In this situation, naïve animals have 
no evolutionary contact with the invasive species and, therefore, lack appropriate innate recognition and responses.

Chemosensory abilities are particularly important for detecting predators in the aquatic medium (Brown et al. 2011). Prey can detect the presence of a particular predator even when it cannot be seen and as a result, display appropriate anti-predator behaviour (Darwish et al. 2005). These chemical cues can take multiple forms: they may be derived directly from the predator themselves or produced via disturbance or mechanical damage to conspecifics (Pfeiffer 1977; Wisenden 2000). In some species, the response is directly proportional to the concentration of the chemical cue, whereas in others there appears to be a critical threshold which must be reached before any response is initiated (Roh et al. 2004). The antipredator response of fathead minnows (Pimephales promelas), for example, is dose specific because their opercula beat rate increases as the scent concentration of predatory pike (Esox lucius) increases (Kusch et al. 2004). In natural circumstances, the concentration of the predator's smell most likely relates to the immediacy of predator attack.

Like fish, many aquatic crustaceans rely on chemoreception to detect predators and prey in the environment. Aquatic crustaceans feature antennae and antennules which possess powerful chemoreception capabilities (Carr and Gurin 1975; Ache et al. 1976). Shrimp antennular filaments can successfully detect chemical substances relating to food resources and predator presence (Ache and Case 1969). It is this adaptation that allows them to successfully manoeuvre to obtain food and elude predators, especially in those species where vision is poorly developed. In mysid shrimp, for example, the introduction of chemical predator cues together with visual signals instigated predator-avoidance behaviour (Lindén et al. 2003). Crowl and Covich (1994) established experimentally that freshwater shrimp express avoidance responses to predator chemical cues, which were amplified in the presence of mechanical cues. Shrimp are also capable of assessing predation risk and modulating their responses depending on the social context (Lindén 2007). Although this limited body of work has shown that shrimp are quite capable of predator voidance, to date, there has been no investigation into the potential role of learning in the development of their avoidance response.

The glass shrimp (Paratya australiensisis) is widely distributed throughout eastern Australia (Richardson et al. 2004) in freshwaters and estuaries (Walsh and Mitchell 1995; Hancock and Bunn 1999). They are an important food source for native aquatic animals and play an important role in ecosystem processes via nutrient recycling (Tamaki et al. 1997; March et al. 2001). Moreover, shrimp strongly influence the composition and distribution of algal and invertebrate assemblages (Pringle 1996; March et al. 2001, 2002). Thus, factors that strongly influence the viability of shrimp populations are likely to have secondary impacts on freshwater ecosystem. Despite its broad distribution and important ecological role, few studies examining the behaviour of glass shrimp have been conducted.

The mosquitofish (Gambusia holbrooki) is a listed pest species around the world and a notable predator of many native species. It was introduced to Australia in the early 1920s in an attempt to control mosquitoes (Lloyd and Tomasov 1985) and within a decade had spread across the entire continent (Arthington 1991). Since its introduction to Australia, Gambusia has been recognised as a key threat to aquatic communities and ecosystems. For example, it has been observed to chase and nip endangered native fish (Keller and Brown 2008) and frogs (Pyke and White 2000). Similarly, the introduction of Gambusia has been directly implicated in the demise of the California fairy shrimp (Linderiella occidentalis) (Leyse et al. 2004). Gambusia is a generalist predator and is active primarily during the day. Its diet is extremely broad but includes a variety of freshwater crustaceans including glass shrimp. The distribution of both species overlaps considerably and as Gambusia continues to colonise new habitats, they will often encounter naïve populations of native shrimp. Little is known about how Gambusia interacts with any Australian freshwater shrimp. 


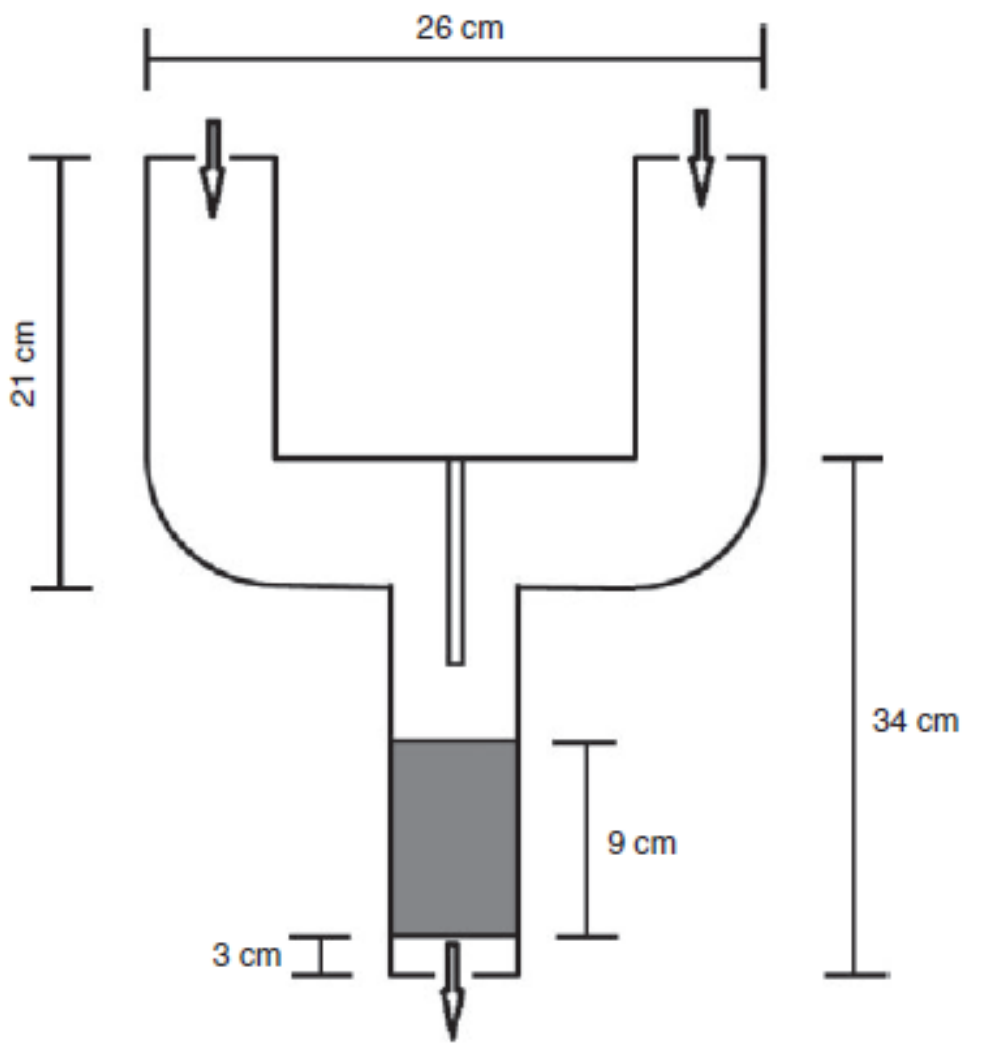

Fig. 1. The Y-maze used to observe behavioural decisions of glass shrimp (Paratya australiensis). Grey area indicates mesh covering where shrimp were inserted. Centre rectangle represents dividing wall, aiding in distinguishing the direction of the scents from the two arms. Flow of water is indicated by the arrows.

Here we examined the interactions between nai"ve glass shrimp and Gambusia to test two specific hypotheses. (1) Can glass shrimp learn to recognise Gambusia as a predator using chemical cues following exposure? (2) Do glass shrimp alter their activity patterns to avoid predatory encounters with Gambusia? We predicted that shrimp would learn to associate the smell of Gambusia with constant harassment and subsequently display an appropriate anti-predator response upon re-exposure. Moreover, when given the opportunity to interact directly with Gambusia, we predicted that the glass shrimp would become nocturnal because Gambusia are inactive at night.

\section{Materials and methods}

\section{Subjects}

Glass shrimp (Paratya australiensisis) (15-20mm in length) raised in earthen ponds devoid of fish predators including Gambusia for multiple generations were purchased from a commercial supplier. After transportation to Macquarie University, they were housed in aquarium $(181 \times 35 \mathrm{~cm})$ containing aged tap water at room temperature $\left(\sim 22^{\circ} \mathrm{C}\right)$.

Thirty Gambusia holbrooki were collected from the Macquarie University Lake and housed in a separate tank of the same size as that housed the glass shrimp. The water temperature within the Gambusia tank was set to $26^{\circ} \mathrm{C}$ to increase the rate of metabolic activity, creating a more pungent scent throughout the 
water. Both shrimp and Gambusia were kept on a 12-h light/dark photoperiod (lights on at 0600 hours) and fed commercial flake food (tetramin tropical flakes, Tetra, Germany) once daily.

\section{Experiment 1: Can glass shrimp learn to recognise Gambusia as a predator using chemical cues following exposure?}

The first experiment was performed in a darkened room containing low light from fluorescent tubes placed evenly around the room. Individual glass shrimp were captured and placed in a bucket of tank water and allowed to settle for $10 \mathrm{~min}$. Females carrying eggs were not included in the sample but no attempt was made otherwise to sex the test subjects.

A small Y-maze ( $6 \mathrm{~cm}$ wide by $50 \mathrm{~cm}$ long) constructed of halved PVC tubing was used to examine glass shrimp behavior (Fig. 1). The maze was placed on a table and filled with aged tap water at room temperature $\left(21^{\circ} \mathrm{C}\right)$. Individual shrimp were then gently scooped out of the bucket using a small cup and placed at the start of the maze behind a physical divider and allowed 4 min to settle into their new environment. After the 4-min interval, $100 \mathrm{~mL}$ of either aged water or Gambusia-scented water (collected directly from a tank in which Gambusia had been held for 7 days) was inserted through a drip into each arm of the maze simultaneously. This was followed by a further $100 \mathrm{~mL}$ of aged water to flush the lines. The arm chosen to contain the Gambusia-scented water was randomised for each replicate. The addition of water to the top of the Y-maze displaced water in the maze which then flowed out the far end. One minute was given to allow the water to be inserted and move down the arm to the divider. The diffusion timing was determined through prior tests using food dyes, which showed that although the flow in the maze was not laminar, the water coming from each arm remained separated in the choice compartment for $\sim 5 \mathrm{~min}$. After this time, the dye from each arm began to diffuse throughout the maze. One minute after the water was added the glass shrimp was released by remotely removing the divider and allowed to navigate the Y-maze. Time taken to make an initial move into the maze and the arm chosen were recorded. The latency to enter the maze was deliberately chosen as a response variable as it provides an indication of how long the shrimp remained still (a typical anti-predator response for cryptic prey). Shrimp were deemed to have made a choice when they moved past the bend and entered one of the arms. This method was repeated for 60 shrimp. The Y-maze was rinsed with tap water before re-setting between replicates. This test examined a possible innate response to predator chemical cues.

After each glass shrimp had completed the initial experiment, it was collected and placed in an individual holding chamber (10 cm diameter and $10 \mathrm{~cm}$ deep). The holding chambers were made from open-ended cylindrical plastic with fly screen covering one end and styrofoam on the other for buoyancy. After being placed into a holder, the holders were numbered and inserted into either the tank containing the Gambusia (exposed treatment) or an empty tank (control treatment) of the same size containing aged tap water. Shrimp in the exposed treatment were frequently harassed by the Gambusia although they could not be killed because of the protective mesh.

Glass shrimp were left in their holders for 3 days during which time they were fed flake food. The shrimp (30 exposed and 30 control) were then reassessed in regards to their preference to Gambusia scent using the same experimental protocol as outlined above and in the same order. The time taken to move into one of the arms and the arm chosen were recorded once again. This second test determined whether the shrimp changed their behaviour in the Y-maze following exposure to the Gambusia. 


\section{Experiment 2: Do glass shrimp alter their activity patterns to avoid predatory encounters with Gambusia?}

In the second experiment, a new batch of 48 glass shrimp (mean length $20 \mathrm{~mm}$ ) was transported to the laboratory, housed at room temperature $\left(22^{\circ} \mathrm{C}\right)$ in groups of three in 16 aquaria $(22.5 \times 34 \mathrm{~cm})$ and allowed to settle for 2 weeks. Water was maintained at $30 \mathrm{~cm}$ and the aquaria were lit by overhead fluorescent lights on a 12-h light/dark photoperiod and furnished with a small bubbler. Lights came on at 0530 hours and off at 1730 hours. Each aquarium contained a refuge (10 long $\times 10$ wide $\times 5$ high $\mathrm{cm})$ surrounded by mesh that was large enough to allow the shrimp access to the hide but excluded the Gambusia. Observations showed that there was ample room under the refuge for all three shrimp to hide at the same time and there were few aggressive interactions. Water chemistry was tested regularly and one-third of the water volume was changed if there was any evidence of ammonia building up in the aquaria. The shrimp and fish were fed small food pellets $(\sim 6 \mathrm{~mm})$ at regular time intervals both day and night to ensure that food was always available. Excess food was siphoned off. A single food pellet was introduced to the aquaria $\sim 10 \mathrm{~cm}$ from the hide. Thus, the shrimp had to emerge from the hide to feed.

Following the 14-day settling period, observations of their activity levels were made every 30 min over 24$\mathrm{h}$ daily cycles for 2 weeks. Observation times and the tanks observed were pseudo-randomised to reduce the number of observations to a reasonable level. This was achieved by assigning each tank four different observation times which shifted by 30 min each day. Thus, each tank was observed at least four times a day resulting in a minimum of 56 observations per tank (more than 840 total observations). Observations at night were conducted under red lights so as to not disturb the shrimp or fish. The behaviour of the animals did not appear to be affected by the presence of an observer at any time.

After 2 weeks of initial observations, a single large, female Gambusia ( $40 \mathrm{~mm}$ standard length) was introduced to the half of the aquaria. We used females simply because they are larger than males and readily attack glass shrimp. The remaining aquaria acted as control treatments. After 3 days of interactions between the Gambusia and shrimp, the observations of shrimp activity resumed for another 2 weeks during which time the Gambusia remained in place in the test treatment.

Data analysis

A $X^{2}$-test was performed to compare $Y$-maze arm choice made by each glass shrimp before and after exposure to Gambusia in the first experiment. The data on the time to enter the Y-maze was skewed and could not be normalised by transformation. Thus, we analysed the data using Wilcoxon signed rank tests. In the second experiment, the number of shrimp feeding before the introduction of Gambusia was subtracted from the number of shrimp feeding after the introduction of Gambusia. The data were checked for normality and the change in feeding activity was analysed using a repeated-measures ANOVA with day and night as the repeated-measure and treatment as a fixed factor. The observations of daily activity patterns were averaged across the two 2-week periods before and after the introduction of Gambusia. All statistics were conducted with Statview 5.01 (SAS Institue, Cary, NC, USA).

\section{Results}

\section{Experiment 1}

Half of both treatment (15/30) and control shrimp (14/30) avoided the Gambusia-scented arm before exposure, as would be expected if the shrimp were choosing an arm at random. Following exposure, 16 of the Gambusia-exposed shrimp and 13 of the control shrimp avoided the Gambusia-scented arm. Glass shrimp had no significant preference for either Gambusia-scented water or aged water before or after exposure and there was no difference between treatments $\left(X^{2}=0.058, P=0.809\right)$. 
There was no significant difference between the time taken by exposed shrimp and control shrimp to choose an arm before exposure to Gambusia (Wilcoxon signed rank test, $Z=0.401, P=0.688$ ). Shrimp in both treatment groups remained motionless (a typical anti-predator response) for the same amount of time ( $180 \mathrm{~s})$ before moving into one of the arms of the maze. After exposure, however, shrimp exposed to Gambusia increased their time to first movement into the Y-maze arms to $320 \mathrm{~s}$, whereas control shrimp showed no change in their latency to enter the maze (Wilcoxon signed rank test, $Z=2.869, P=$ 0.004; Fig. 2).

\section{Shrimp leam to avoid an invasive fish species}

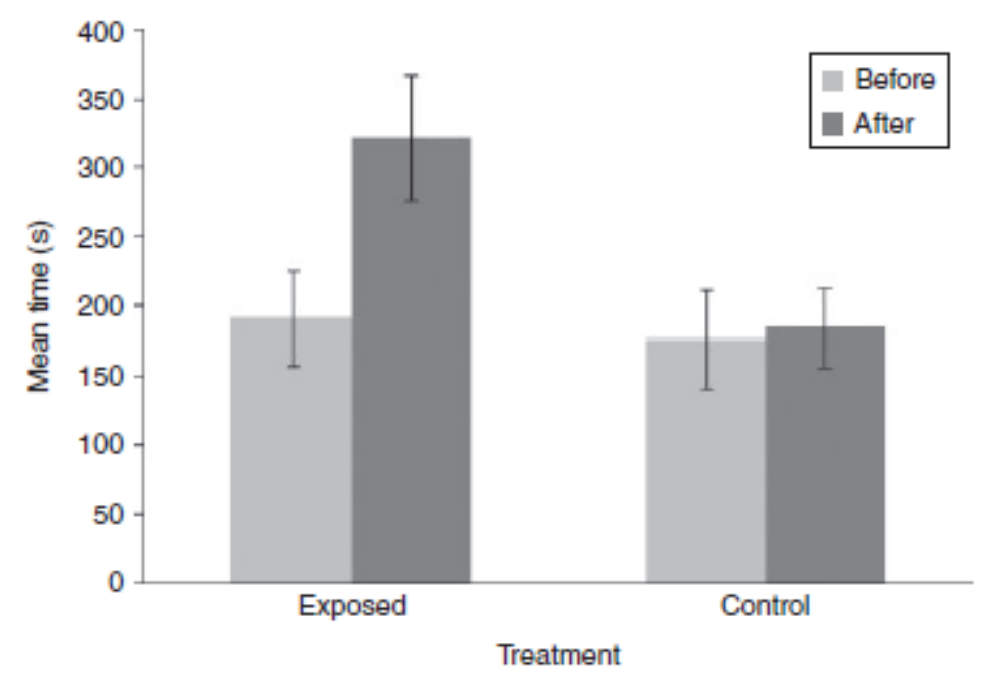

Fig. 2. The mean ( \pm s.e.) time taken by glass shrimp (Paratya australiensis) exposed to Gambusia and not exposed (control) to move through the Y-maze before exposure (light bars) and after exposure (dark bars).

\section{Experiment 2}

Shrimp in both the control and exposed treatments showed similar foraging activities before the introduction of Gambusia. Most of the activity was concentrated during daylight hours from 0530 hours until 1730 hours (Fig. 3a). Once the Gambusia were added to the test treatment, the shrimp showed a significant shift in foraging activity to the night whereas no shift was observed in the control treatment (Fig. 3b). The change in activity levels from before to after the introduction of Gambusia revealed a significant interaction between treatment and time period (repeated-measures ANOVA, $F_{1,14}=228.93, P<0.001$ ). The number of shrimp feeding during the day in the second time period increased in the absence of Gambusia (mean \pm s.e.: $0.7 \pm 0.06$ to $1.1 \pm 0.07$ ) whereas they decreased in the presence of Gambusia ( $1.0 \pm 0.07$ to $0.3 \pm 0.04)$. Similarly, the number of shrimp feeding in the night showed no change in the treatment lacking Gambusia ( $0 \pm 0.01$ to $0.1 \pm 0.02)$ but increased dramatically in the treatment with Gambusia (0.1 \pm 0.02 to $1.4 \pm 0.13$ ). In the Gambusia-exposed treatment, shrimp were constantly harassed and seven were killed and eaten by Gambusia in the early days of the exposure period. This means that the increase in night time foraging activity is actually conservative in this treatment. Our observations also indicated that Gambusia were almost completely inactive during the night, spending most of their time drifting near the surface of the water. 


\section{Discussion}

Our results show that glass shrimp are capable of adjusting their behaviour as a result of experience and learn to recognise and subsequently avoid a novel predator. Although shrimp with prior experience of Gambusia did not avoid the Y-maze arm containing Gambusia cues, they did show anti-predator behaviour in the form of a 'freeze' response. Shrimp with no prior exposure, in contrast, showed no change in behaviour in response to chemical cues emanating from the predator. When the shrimp and Gambusia were allowed to interact directly, naïve shrimp shifted their foraging activity from diurnal to nocturnal foraging when the Gambusia were not active. These results clearly indicate that glass shrimp can learn to recognize novel, invasive predators using their chemosensory capabilities alone. When given limited escape choices, shrimp decreased their activity levels to avoid detection, but over the longer term they adjusted their temporal activity patterns to reduce the probability of encountering the novel predator.

\section{Do glass shrimp learn to recognise Gambusia scent?}

After exposure to Gambusia, glass shrimp delayed making a choice between the predator scent and aged water. Rather than moving into one of the arms following a short delay, as they had done before exposure, after exposure to Gambusia shrimp remained stationary underneath the protective mesh. Indeed, the time to emerge from the sheltered start area increased from $180 \mathrm{~s}$ before exposure to $320 \mathrm{~s}$ after exposure to Gambusia. The control shrimp, having not been exposed to Gambusia, showed no such change in their behaviour. The most parsimonious explanation for this result is that the shrimp made an association between the smell of the Gambusia and fear or stress created by being continuously attacked during the 3 days of exposure, causing them to behave cautiously when they detected the scent of Gambusia in the maze. Whether that learning response occurred very early on remains to be seen, although one trial learning has been observed in predatory contexts in a variety of taxa (Csanyi and Doka 1993; Griffin 2008). Given the nature of the predator-prey interaction here, where the prey item is rather large, it is likely that shrimp in the wild would survive an initial attack from Gambusia, or indeed multiple attacks, providing ample opportunity for learning to develop.

It might be argued that the Gambusia-exposed shrimp were not responding directly to the scent of Gambusia but simply showed a generalised higher level of caution when placed back in the maze as a result of heightened fear or stress levels. In either scenario, it is clear that their response would be the most appropriate action to take having been previously exposed to danger. To remain safe, glass shrimp minimised movement to avoid detection by the predator and potentially to maximize distance from the cue source. It is known that many aquatic predators rely on movement as key stimuli for prey detection and given that glass shrimp are almost completely transparent, freezing is likely to be an effective antipredator response. Related studies have observed similar behaviour where prey reduced their rates of feeding and locomotion in the presence of a predator cue (Hazlett et al. 2002; Acquistapace et al. 2003). Lindén et al. (2003), for example, assessed anti-predator behavior in two mysid shrimp species and found that the mysid Neomysis integer reduced swimming and remained stationary in the presence of predator cues. In comparison, Praunus flexuosus hid in vegetation. Thus, it appears that many shrimp rely on crypsis to avoid detection by predators.

Although it is clear that glass shrimp displayed a freezing behaviour when predator chemical cues were detected, it is still puzzling why they showed no avoidance of the maze arm containing the predator odour once they did begin to move. It may be that the shrimp detected that the scent was coming from upstream but did not differentiate between the left and right arms of the maze. Alternatively, because the exposed glass shrimp waited over 5 min before entering the maze after exposure, the Gambusia scent may have been diluted sufficiently enough to signal a reduction in danger. Our prior tests examining diffusion within the maze using food dye suggest that this is likely. Once dilution to low concentrations had occurred, anti- 
predator behaviour gave way to normal exploratory behaviour. This response has been observed in previous studies where little or no anti-predator behaviour is seen when the predator scent is heavily diluted (Dupuch et al. 2004). It may be pertinent in future experiments to allow shrimp to move further away from the scent and to generate continuous laminar flow to prevent cue diffusion by modifying the apparatus and experimental approach.
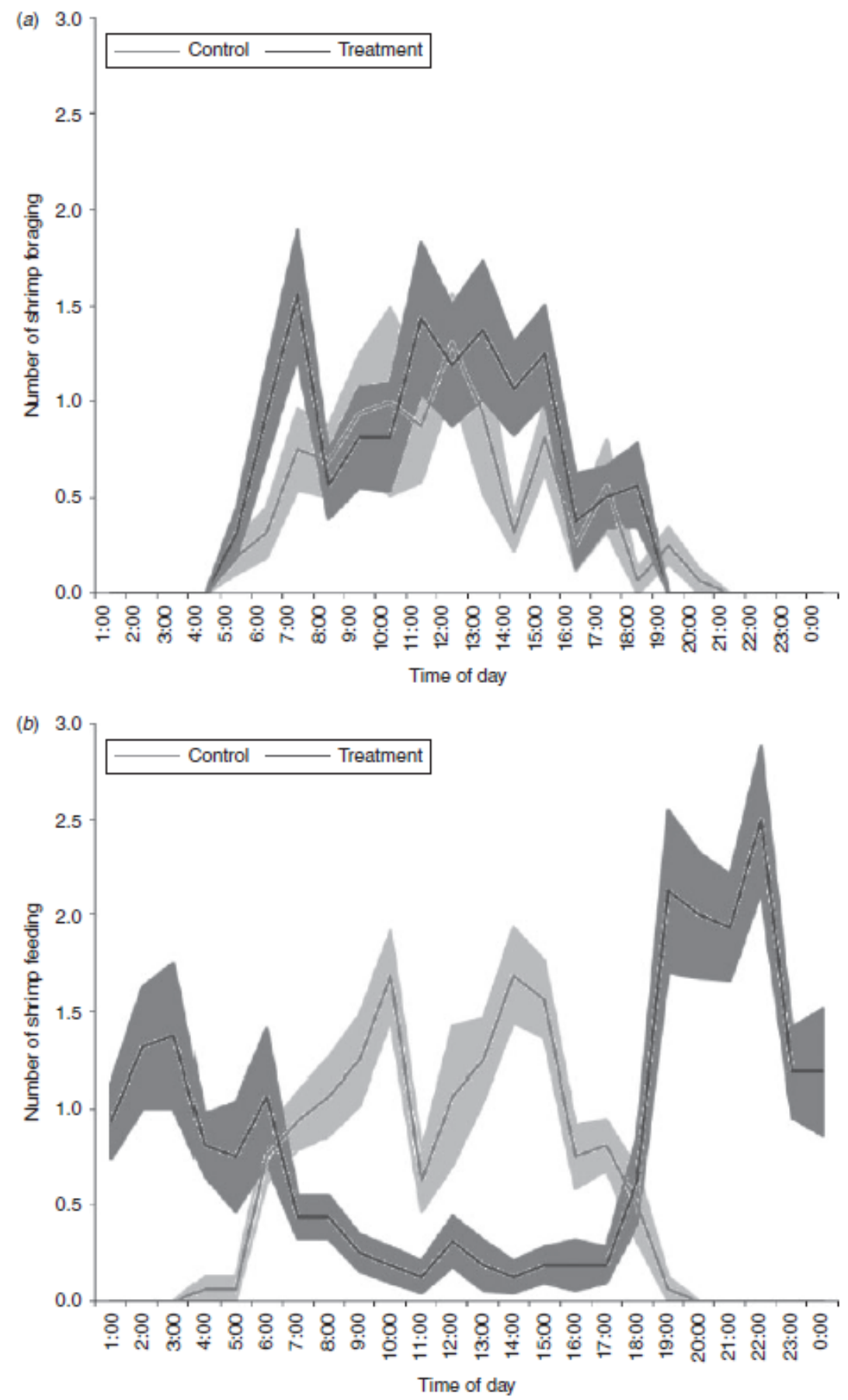

Fig. 3. The average number of glass shrimp feeding over a 24-h period (a) before the introduction of Gambusia and $(b)$ after the introduction of Gambusia. Light lines represent the mean for the control fish and the dark lines represent the mean for fish exposed to Gambusia. Filled areas around the lines represent s.e. 
Lima and Bednekoff (1999) formulated a risk assessment model which incorporates temporal stability of predation risk. The model suggests that animals exposed to brief and infrequent bouts of predation are likely to show the most intense antipredator responses (i.e. they show the greatest loss in foraging opportunity in favour of anti-predator behaviour). Somewhat paradoxically, however, animals constantly exposed to highly levels of risk show moderate rates of foraging behaviour to counteract lost foraging opportunities. Due to the length of time that glass shrimp in the exposed treatment spent in constant contact with Gambusia, it may well be that the shrimp wait for some period to assess the risk, perhaps waiting for direct contact or confirmation via alternative cues (e.g. visual or mechanical), and then emerge to forage if no negative feedback is forth coming even though they can still detect the predator scent. Many studies on learned predator recognition have included visual cues alongside chemical cues. It is often observed that chemical cues and visual cues alone provided sufficient information in isolation to initiate anti-predator responses. However, when paired together, the response can be additive (Crowl and Covich 1994; Lindén et al. 2003). Future study on glass shrimp should examine the effects of visual reinforcement in combination with chemical cues.

\section{Do glass shrimp adjust their activity patters to avoid Gambusia?}

When glass shrimp and Gambusia were placed in aquaria together, the shrimp showed a complete switch in their activity patterns. Before exposure, the shrimp fed almost entirely during the day, but once Gambusia was introduced they completely switched to nocturnal feeding (Fig. 3). Our casual observations of the behaviour of Gambusia showed that they were most likely sleeping during the night, thus, the shrimp made the most of this period to emerge from cover and forage in relative safety.

When taken together, our results suggest that when Gambusia come into contact with naïve populations of glass shrimp, there are several possible scenarios. First, frequent attacks by Gambusia would most likely lead to enhanced anti-predator behaviour and reduced foraging opportunities, perhaps leading to population extirpation (Howe et al. 1997; Warburton and Madden 2003). Alternatively, glass shrimp may learn to coexist with Gambusia. Constant attack from Gambusia would promote glass shrimp to display niche shifts in order to survive, a behaviour that has been illustrated in other native species (Keller and Brown 2008). The results of our second experiment clearly show that shrimp are capable of altering their daily activity patterns to avoid contact with the invasive pest species. In the presence of Gambusia, glass shrimp only emerge at night when the Gambusia are inactive and thereby avoid predation. Although our experiments did not offer refuge in three dimensions, it is also possible for shrimp to switch to benthic feeding to avoid contact with Gambusia which show a strong preference for surface feeding (Pen et al. 1993; Rinco'n et al. 2002; Keller and Brown 2008). For example, native Hawaiian shrimp (Halocaridina rubra) in anchialine pools undergo diel migrations to avoid predation by introduced Gambusia (Carey et al. 2011).

In conclusion, although it is clear Gambusia can predate upon and harass glass shrimp, our data show that shrimp can learn to recognise novel predators and instigate an appropriate anti-predator response on subsequent exposure in the short term and shift their daily activity patterns over the longer term. When taken together with previous research, the data suggest that learning to recognise predators in aquatic crustaceans can occur relatively quickly and their learning abilities appear to be on par with fish (Hazlett et al. 2002). In the present context, this behavioural plasticity provides an avenue for their coexistence with introduced novel predators. Further studies could examine the behaviour of shrimp in semi-natural conditions and the potential fitness consequences following the introduction of Gambusia. Such interactions are likely to shape the entire aquatic ecosystem since shrimp occupy a keystone position in the food web of many freshwater environments (Pringle 1996; March et al. 2001). 


\section{Acknowledgements}

Thanks to Macquarie University for providing funds, equipment and laboratory space. CB was supported by an Australian Research Fellowship. This work was conducted with permission from the Macquarie University Ethics Committee (ARA 2010/050). We also thank the anonymous referees for their helpful comments.

\section{References}

Ache, A., and Case, J. (1969). An analysis of antennular chemoreception in two commensal shrimps of the genus Betaeus. Physiological Zoology 42, 361-371.

Ache, B. W., Fuzessery, Z. M., and Carr, W. E. S. (1976). Antennular chemosensitivity in the spiny lobster, Panulirus argus: comparative tests of high and low molecular weight stimulants. The Biological Bulletin 151, 273-282. doi:10.2307/1540660

Acquistapace, P., Hazlett, B. A., and Gherardi, F. (2003). Unsuccessful predation and learning of predator cues by crayfish. Journal of Crustacean Biology 23, 364-370. doi:10.1651/02780372(2003)023[0364:UPALOP]2.0.CO;2

Arthington, A. H. (1991). Ecological and genetic impacts of introduced and translocated freshwater fishes in Australia. Canadian Journal of Fisheries and Aquatic Sciences 48, 33-43. doi:10.1139/F91-302

Brown, G. E., Ferarri, M. C. O., and Chivers, D. P. (2011). Learning about danger: chemical alarm cues and threat sensitive assessment of predation risk by fishes. In 'Fish Cognition and Behaviour, 2nd edition'. (Eds C. Brown, K. Laland and J. Krause.) pp. 59-80. (Blackwell: Oxford.)

Carey, C., Ching, M., Collins, S., Early, A., Fetzer, W., Chai, D., and Hairston, N. (2011). Predatordependent diel migration by Halocaridina rubra shrimp (Malacostraca: Atyidae) in Hawaiian anchialine pools. Aquatic Ecology 45, 35-41. doi:10.1007/S10452-010-9321-0

Carr, W. E. S., and Gurin, S. (1975). Chemoreception in the shrimp, Palaemonetes pugio: comparative study of stimulatory substances in human serum. The Biological Bulletin 148, 380-392. doi:10.2307/1540515

Crowl, T. A., and Covich, A. P. (1994). Responses of freshwater shrimp to chemical and tactile stimuli from a large decapod predator. Journal of the North American Benthological Society 13, 291298. doi:10.2307/1467247

Csanyi, V., and Doka, A. (1993). Learning interactions between prey and predator fish. Marine Behaviour and Physiology 23, 63-78. doi:10.1080/10236249309378857

Darwish, T. L., Mirza, R. S., Leduc, A. O. H. C., and Brown, G. E. (2005). Acquired recognition of novel predator odour cocktails by juvenile glowlight tetras. Animal Behaviour 70, 83-89. doi:10.1016/J.ANBEHAV.2004.09.017

Dupuch, A., Magnan, P., and Dill, L. M. (2004). Sensitivity of northern redbelly dace, Phoxinus eos, to chemical alarm cues. Canadian Journal of Zoology 82, 407-415. doi:10.1139/Z04-003

Griffin, A. S. (2008). Socially acquired predator avoidance: is it just classical conditioning? Brain Research Bulletin 76, 264-271. doi:10.1016/J.BRAINRESBULL.2008.02.005

Hancock, M. A., and Bunn, S. E. (1999). Swimming response to water current in Paratya australiensis Kemp, 1917 (Decapoda, Atyidae) under laboratory conditions. Crustaceana 72, 313-323. doi:10.1163/156854099503393

Hazlett, B. A., Acquistapace, P., and Gherardi, F. (2002). Differences in memory capabilities in invasive and native crayfish. Journal of Crustacean Biology 22, 439-448. doi:10.1651/02780372(2002)022[0439:DIMCII]2.0.CO;2 
Howe, E., Howe, C., Lim, R., and Burchett, M. (1997). Impact of the introduced poeciliid Gambusia holbrooki (Girard, 1959) on the growth and reproduction of Pseudomugil signifer (Kner, 1865) in Australia. Marine and Freshwater Research 48, 425-434. doi:10.1071/MF96114

Keller, K., and Brown, C. (2008). Behavioural interactions between the introduced plague minnow Gambusia holbrooki and the vulnerable native Australian ornate rainbowfish Rhadinocentrus ornatus, under experimental conditions. Journal of Fish Biology 73, 1714-1729. doi:10.1111/J.1095-8649.2008.02045.X

Kelley, J. L., and Magurran, A. E. (2011). Learned defences and counter defences in predator-prey interactions. In 'Fish Cognition and Behaviour, 2nd edition' (Eds C. Brown, K. Laland and J. Krause.) pp. 36-58. (Blackwell: Oxford.)

Kusch, R. C., Mirza, R. S., and Chivers, D. P. (2004). Making sense of predator scents: investigating the sophistication of predator assessment abilities of fathead minnows. Behavioral Ecology and Sociobiology 55, 551-555. doi:10.1007/S00265-003-0743-8

Leyse, K. E., Lawler, S. P., and Strange, T. (2004). Effects of an alien fish, Gambusia affinis, on an endemic California fairy shrimp, Linderiella occidentalis: implications for conservation of diversity in fishless waters. Biological Conservation 118, 57-65. doi:10.1016/J.BIOCON.2003.07.008

Lima, S. L., and Bednekoff, P. A. (1999). Temporal variation in danger drives antipredator behaviour: the predation risk allocation hypothesis. American Naturalist 153, 649-659. doi:10.1086/303202

Lindén, E. (2007). The more the merrier: swarming as an antipredator strategy in the mysid Neomysis integer. Aquatic Ecology 41, 299-307. doi:10.1007/S10452-006-9055-1

Lindén, E., Lehtiniemi, M., and Viitasalo, M. (2003). Predator avoidance behaviour of Baltic littoral mysids Neomysis integer and Praunus flexuosus. Marine Biology 143, 845-850. doi:10.1007/S00227003-1149-X

Lloyd, L. N., and Tomasov, J. F. (1985). Taxonomic status of the mosquitofish, Gambusia affinis (Poeciliidae), in Australia. Australian Journal of Marine and Freshwater Research 36, 447-451. doi:10.1071/MF9850447

Magurran, A. E. (1990). The inheritance and development of minnow antipredator behaviour. Animal Behaviour 39, 834-842. doi:10.1016/S0003-3472(05)80947-9

Magurran, A. E., and Seghers, B. H. (1990). Population differences in the schooling behaviour of newborn guppies, Poecilia reticulata. Ethology 84, 334-342. doi:10.1111/J.1439-0310.1990.TB00807.X

March, J. G., Benstead, J. P., Pringle, C. M., and Ruebel, M. W. (2001). Linking shrimp assemblages with rates of detrital processing along an elevational gradient in a tropical stream. Canadian Journal of Fisheries and Aquatic Sciences 58, 470-478. doi:10.1139/F00-263

March, J. G., Pringle, C. M., Townsend, M. T., and Wilson, A. I. (2002). Effects of freshwater shrimp on assemblages of benthic communities along an altitudinal gradient of a tropic island stream. Freshwater Biology 47, 377-390. doi:10.1046/J.1365-2427.2002.00808.X

Pen, L. J., Potter, I. C., and Calver, M. C. (1993). Comparisons of the food niches of three native and two introduced fish species in an Australian river. Environmental Biology of Fishes 36, 167-182. doi:10.1007/BF00002797

Pfeiffer, W. (1977). The distribution of fright reaction and alarm substance cells in fishes. Copeia 1977, 653-665. doi:10.2307/1443164

Pringle, C. M. (1996). Atyid shrimps (Decapoda: Atyidae) influence the spatial heterogeneity of the algal communities over different scales in tropical montane streams, Puerto Rico. Freshwater Biology 35, 125-140. doi:10.1046/J.1365-2427.1996.00487.X

Pyke, G. H., and White, A. W. (2000). Factors influencing predation on eggs and tadpoles of the endangered green and golden bell frog Litorea aurea by the introduced plague minnow Gambusia holbrooki. Australian Zoologist 31, 496-505. 
Richardson, A. J., Growns, J. E., and Cook, R. A. (2004). Distribution and life history of caridean shrimps in regulated lowland rivers in southern Australia. Marine and Freshwater Research 55, 295-308. doi:10.1071/MF03126

Rincón, P. A., Correas, A. M., Morcillo, F., Risueño, P., and Lobón-Cerviá, J. (2002). Interaction between the introduced eastern mosquitofish and two autochthonus Spanish toothcarps. Journal of Fish Biology 61, 1560-1585. doi:10.1111/J.1095-8649.2002.TB02498.X

Roh, E., Mirza, R. S., and Brown, G. E. (2004). Quality or quantity? The role of donor condition in the production of chemical alarm cues in juvenile convict cichlids. Behaviour 141, 1235-1248. doi:10.1163/1568539042729667

Tamaki, A., Ingole, B., Ikebe, K., Muramatsu, K., Taka, M., and Tanaka, M. (1997). Life history of the ghost shrimp, Callianassa japonica Ortmann (Decapoda: thalassinidea), on an intertidal sandflat in western Kyushu, Japan. Journal of Experimental Marine Biology and Ecology 210, 223-250. doi:10.1016/S0022-0981(96)02709-8

Walsh, C. J., and Mitchell, B. D. (1995). The freshwater shrimp Paratya australiensis (Kemp, 1917) (Decapoda: Atyidae) in estuaries of southwestern Victoria, Australia. Marine and Freshwater Research 46, 959-965. doi:10.1071/MF9950959

Warburton, K., and Madden, C. (2003). Behavioural responses of two native Australian fish species (Melanotaenia duboulayi and Pseudomugil signifer) to introduced poeciliids (Gambusia holbrooki and Xiphophorus helleri) in controlled conditions. Proceedings of the Linnean Society of New South Wales 124, 115-123.

Wisenden, B. D. (2000). Olfactory assessment of predation risk in the aquatic environment. Philosophical Transactions of the Royal Society of London. Series B, Biological Sciences 355, 1205-1208. doi:10.1098/RSTB.2000.0668 